\title{
Ambient RF Energy Harvesting
}

\author{
D. BOUCHOUICHA ${ }^{1}$, F. DUPONT ${ }^{1}$, M. LATRACH ${ }^{2}$, L.VENTURA ${ }^{3}$ \\ ${ }^{1}$ STMicroelectronics, 16 Rue Pierre et Marie Curie 37071 Tours France \\ dhaou.bouchouicha@st.com,francois.dupont@st.com \\ laurent.ventura@univ-tours.fr \\ ${ }^{2}$ Groupe RF\& Hyperfréquence- école supérieure d'électronique de l'ouest(ESEO) \\ 4 Rue Merlet de la Boulaye, BP 30926, 49009 Angers, France \\ mohamed.latrach@eseo.fr \\ ${ }^{3}$ Laboratoire de microélectronique de puissance-Université de Tours. \\ 16 rue Pierre et Marie Curie 37071 Tours France.
}

\begin{abstract}
:
In this paper, we present a study of ambient RF energy harvesting techniques. The measurement of the ambient RF power density is presented. The average of the density in broadband $(1 \mathrm{GHz}-$ $3.5 \mathrm{GHz})$ is in the order of $-12 \mathrm{dBm} / \mathrm{m}^{2}\left(63 \mu \mathrm{W} / \mathrm{m}^{2}\right)$. Two systems have been studied to recover the RF energy. The first is a broadband system without matching circuit. The second is a narrow band system $(1.8-1.9 \mathrm{GHz})$ with a matching circuit. The rectifier circuit RF / DC and the choice of the load to optimize the DC power recovered are presented.

The preliminary results indicate that the recovered energy is not sufficient to directly power devices but could be stored in a super-capacity or micro-batteries.
\end{abstract}

Keywords - harvesting energy, RF energy, wireless sensor, rectenna.

\section{Introduction:}

In recent years the use of wireless devices is growing in many applications like mobile phones or sensor networks. This increase in wireless applications has generated an increasing use of batteries. Many research teams are working on the autonomy of the batteries by reducing the consumption of the devices. Others teams have chosen to recycle ambient energy like in MEMS [1]. The charging of multiple applications is easy because the user can do it easily, like for mobile phones. But for other applications, like wireless sensor nodes located in difficult access environments, the charging of the batteries remains a major problem. This problem increase when the number of devices is large and are distributed in a wide area or located in inaccessible places. The uses of the Wireless Power Transmission (WPT) allow the overcoming of these problems.

The rectification of microwave signals to DC power has been proposed and researched in the context of high-power beaming since the 1950s [2]. It has been proposed for helicopter powering [3], solar power satellite (SPS) [4], the SHARP System [5], and recently for RFID system.
The principle of this kind of power transfer is presented in the Figure 1.

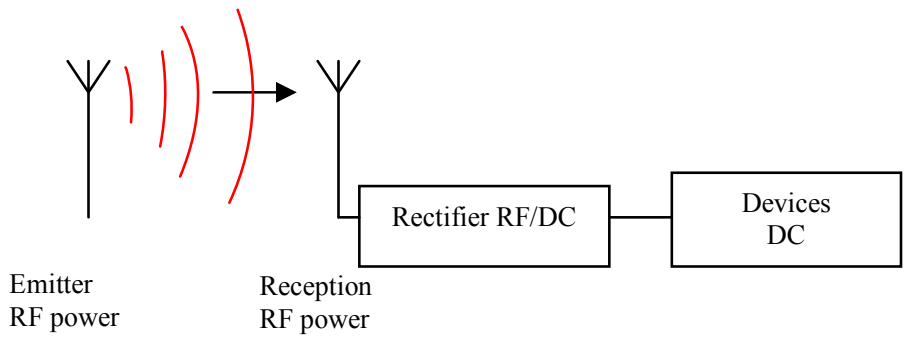

Fig.1. Conceptual view of the WPT system.

In this paper we focus on ambient RF energy. We propose to use the energy from commercial RF broadcasting stations like GSM, TV, WIFI or Radar to supply energy for wireless sensor nodes or other applications. This powering method can be especially interesting for sensor nodes located in remote places, where other energy sources like solar or wind energies are not feasible.

The DC power depends on the available RF power and conversion efficiency RF/DC.

$$
P_{d c}=\eta_{R F / D C} \cdot P_{R F}
$$

The choice of antenna and frequency band is very important to optimize the DC power harvested.

In the section 2 of this paper we present the measurements of the ambient RF power density. The evolution of this density is studied as a function of the frequency and time. The section 3 discusses the design of the broadband rectenna without a matching circuit. The section 4 presents a study of the rectenna with a matching circuit.

\section{Measurements of density RF power:}

Multiple sources of different frequencies are radiating power in all directions in a rich scattering environment (Fig. 2). 


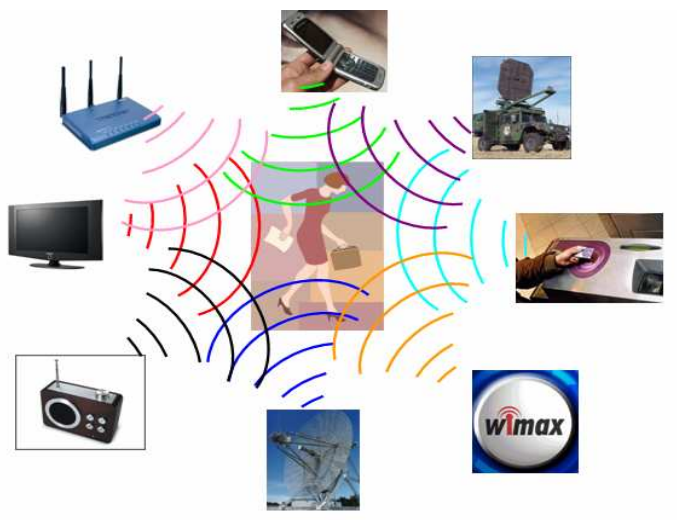

Fig.2. examples of the different radiating sources

We have measured the RF power density in the different points in the urban environments. The variation of this density in $\mathrm{dBm} / \mathrm{m}^{2}$ depends on the frequency and time in the $680 \mathrm{MHz}-3.5 \mathrm{GHz}$ band and is presented in the Fig.3. The power density variation is found to be between $60 \mathrm{dBm} / \mathrm{m}^{2}$ and $-14.5 \mathrm{dBm} / \mathrm{m}^{2}\left(1 \mathrm{nW} / \mathrm{m}^{2}\right.$ and $\left.35.5 \mu \mathrm{W} / \mathrm{m}^{2}\right)$ and is constant over time. The maximum of this power density has been measured in the $1.8 \mathrm{GHz}-1.9 \mathrm{GHz}$ band. The summation of the power density of all the measured signals (Fig.4) provides a greater power density around-

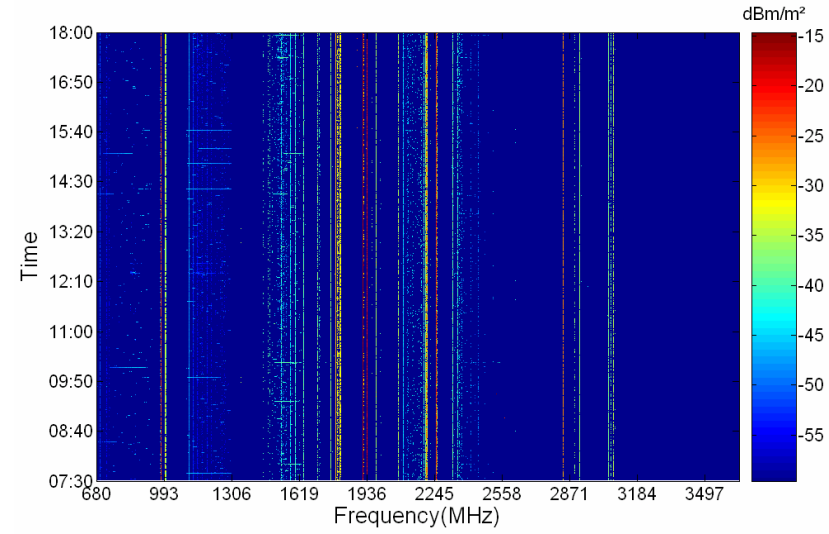

$12 \mathrm{dBm} / \mathrm{m}^{2}$. The RF energy harvesting system principle is presented in the Fig. 5

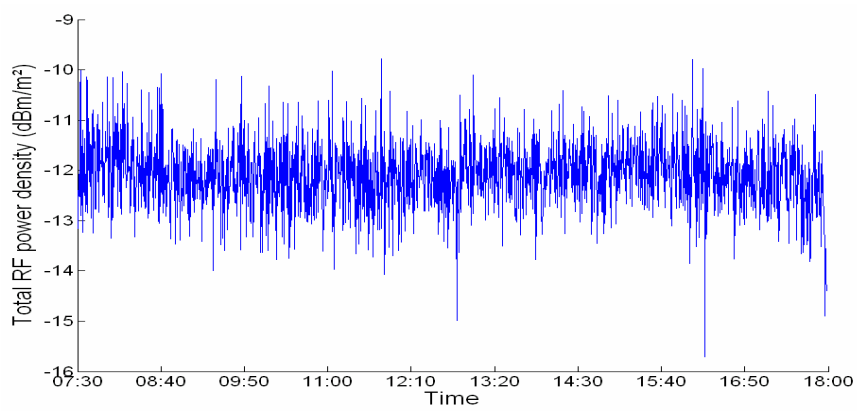

Fig.3 Measured RF power density versus time $(680 \mathrm{MHz}-$ $3500 \mathrm{MHz}$ )

Fig.4. the total RF power density measured versus time

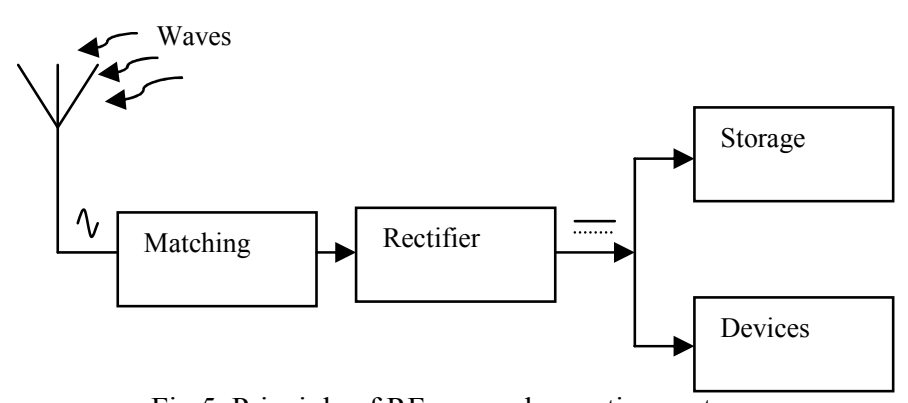

Fig.5. Principle of RF energy harvesting system

\section{Broadband system:}

The broadband system consists of two parts; the rectifier without matching circuit and the broadband antenna. The goal of the system is to maximize the DC power harvested and is designed to recover all signals available. For this issue we must use an omni-directional broadband antenna.

\subsection{Rectifier}

The RF/DC converter is a voltage doubler and has been designed and simulated by using the Advanced Design System (ADS) software, which uses the harmonic-balance method. This circuit is optimized and achieved by using a commercial zero biased Schottky diode HSMS2850 (Fig.6)

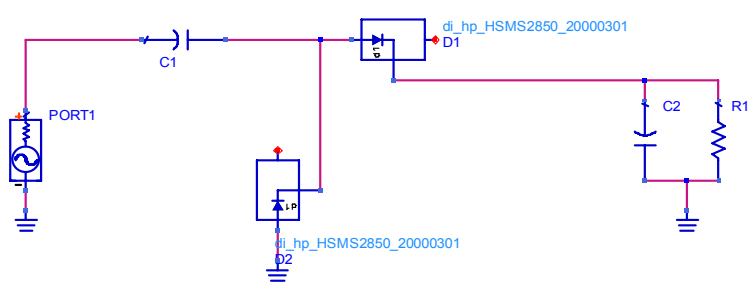

Fig.6: Schema of the rectifier

Fig.7 Show the impedance versus frequency. It is equivalent to a parallel RC circuit. This type of impedance can be adapted for a wide frequency range, but the loss in the matching circuit will be very important [6]. To avoid these losses we connect the antenna directly to the rectifier without a matching circuit.

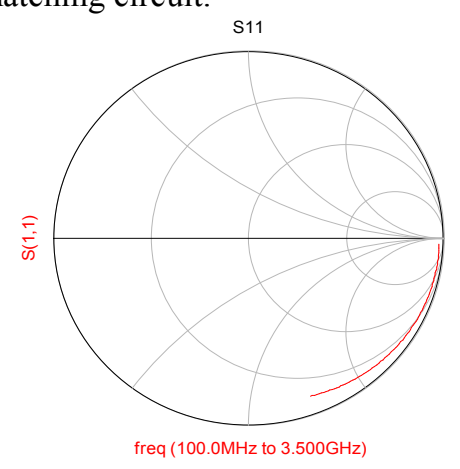

Fig.7: The impedance of rectifier without matching circuit as a function of the frequency 
The fabrication of the microwave rectifier is done by using FR4 as a substrate (relative permittivity 4.4, tangent losses 0.02 , thickness $0.8 \mathrm{~mm}$ ). Fig. 8 shows the variations of the output DC power versus the input RF power at $1.5 \mathrm{GHz}$. The simulation and measurement results are in good agreement for RF power more than $-32 \mathrm{dBm}$.

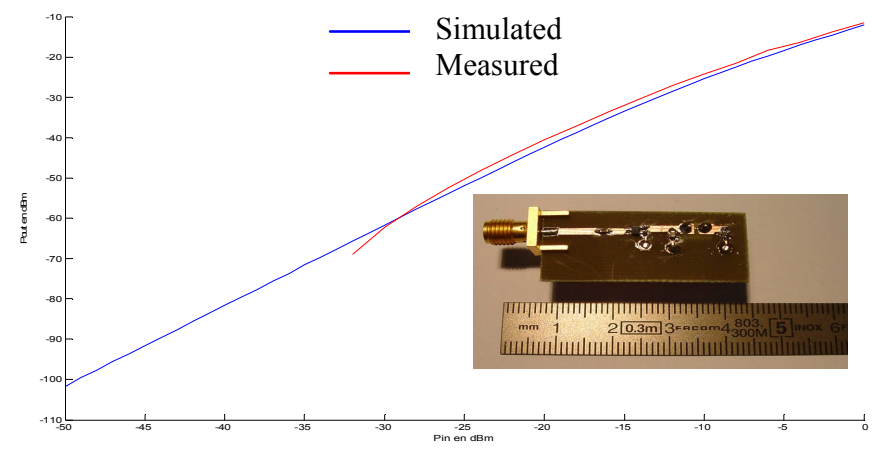

Fig.8: Simulated and measured DC power as a function of RF power $(1.5 \mathrm{GHz})$

The impedance of the antenna affects the DC power recovered. In Fig.9, the DC power as a function of resistance of antenna for a wide band frequency is presented. For resistances lower than $50 \Omega$ the DC power is very low. The optimum resistance value to increase the DC power over the entire frequency range $(1 \mathrm{GHz}-3 \mathrm{GHz})$ is about $100 \Omega$.

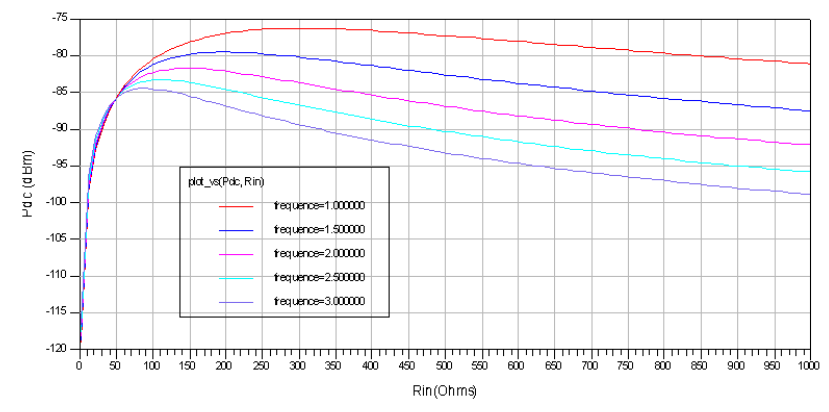

Fig.9: Simulated result of the DC power as a function of the antenna resistance. The input RF power is $\mathrm{Pin}=-40 \mathrm{dBm}$.

\subsection{Spiral Antenna}

As seen in the previous section, the major problem in broad-band rectenna design is linked to the matching circuit. For maximal power transfer, the antenna impedance must be matched to the optimal diode impedance for all frequencies. Our approach is to present a constant impedance of $100 \Omega$ to the diode by using a frequency-independent antenna.

An equiangular spiral with dimensions shown in Fig. 10 was chosen for the following reasons:

1) Uni-planar with convenient feed point for diode connection.

2) Possible dual polarization;

3) Broadband antenna

4) Omni-directional radiation pattern

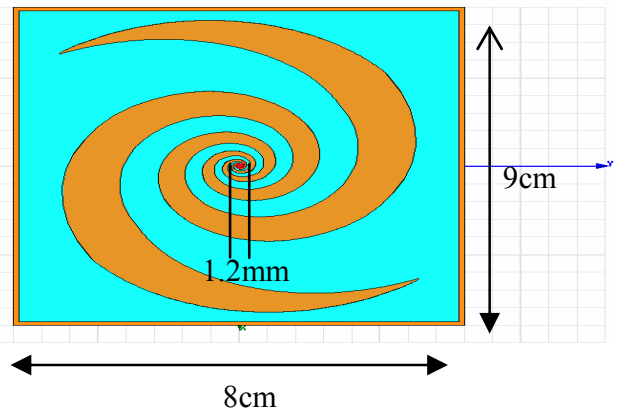

Fig.10 Spiral antenna

The spiral antenna was simulated with HFSS tools [7].As shown in Fig 11, measured and simulated return losses are in good agreement. In all band $(1 \mathrm{GHz}-3 \mathrm{GHz})$ the return loss is lower than $-10 \mathrm{~dB}$.

Fig. 12 shows the radiated antenna energy in the space. The shape of radiation varies according to the frequency. A quasi Omni directional radiation is obtained for low frequencies around $1 \mathrm{GHz}$. In the all frequency band $(1 \mathrm{GHz}-$ $3 \mathrm{GHz}$ ) the gain is more than $2.5 \mathrm{dBi}$ (Fig.13). The gain can reach $7 \mathrm{dBi}$ for a frequency of $3 \mathrm{GHz}$.

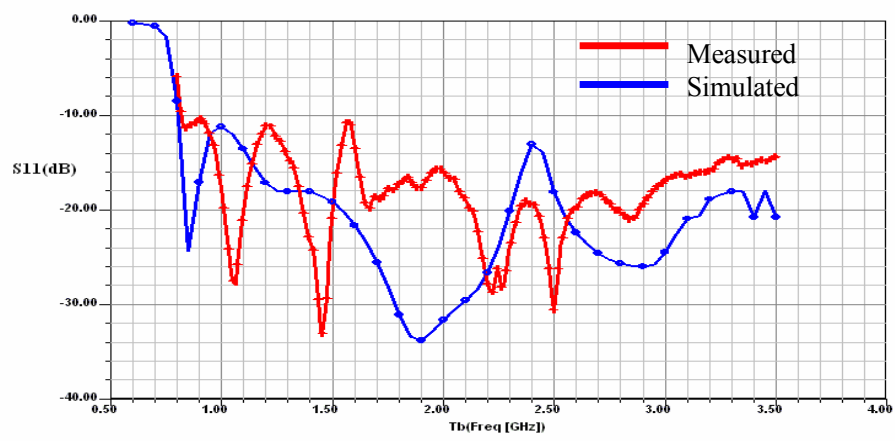

Fig.11. Antenna return loss
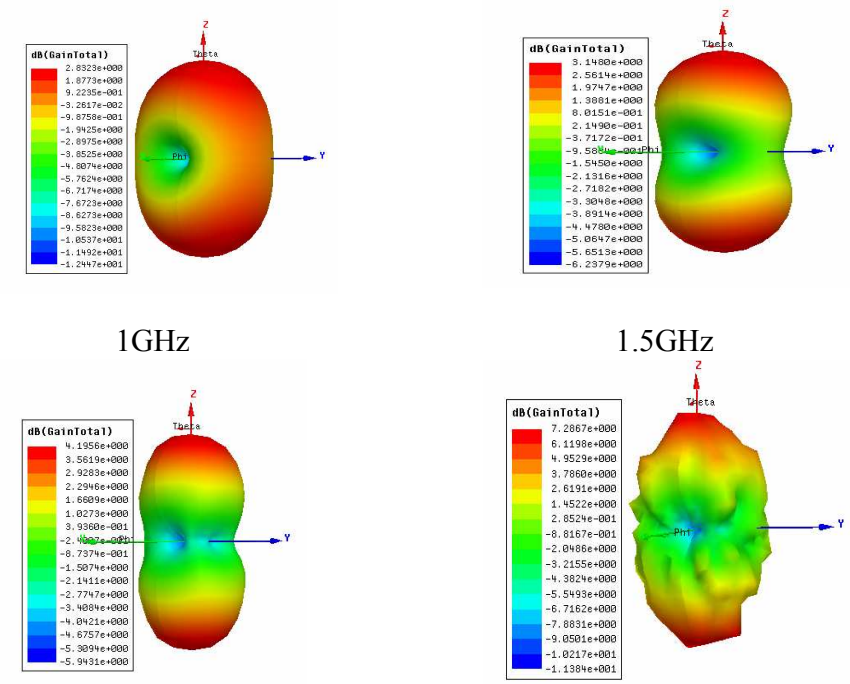

$2 \mathrm{GHz}$

$3 \mathrm{GHz}$

Fig.12: Radiation pattern of spiral antenna. 


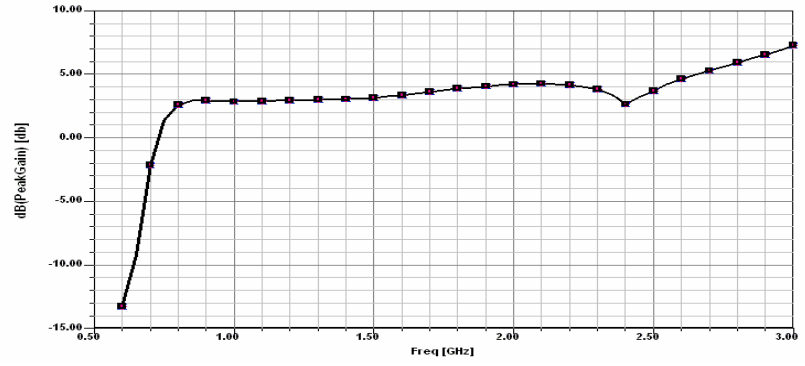

Fig.13 Maximum gain

The Maximum RF power that the spiral antenna can capture in the order of $-42 \mathrm{dBm}(63 \mathrm{nW})$.

In Fig.14, the DC power as a function of load RL for a wide band frequency is presented. The optimum load to maximize the DC power is $18 \mathrm{~K} \Omega$. With this load the DC power is estimated between $5 \mathrm{pW}$ and $10 \mathrm{pW}(-83 \mathrm{dBm}-$ $80 \mathrm{dBm})$. The rectenna is presented in Fig.15. The measured power in environment is around $-79 \mathrm{dBm}$ $(12.5 \mathrm{pW})$. It is in the range of estimated DC power.

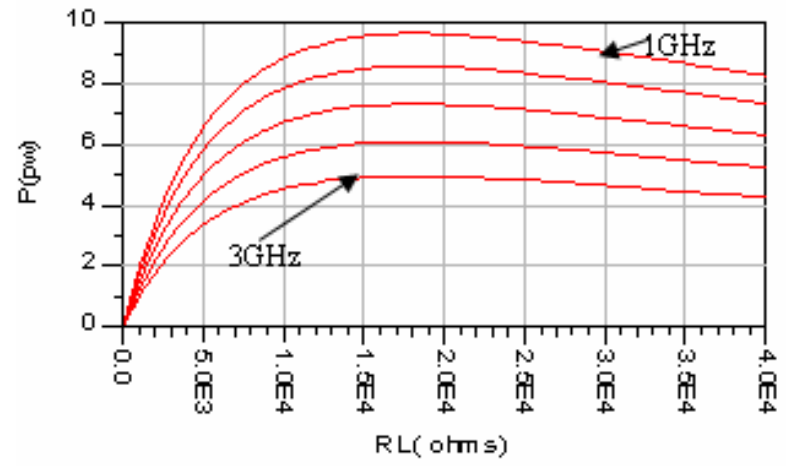

Fig.14: Output power as function of RL load for Pin=-42dBm $(63 n W)$

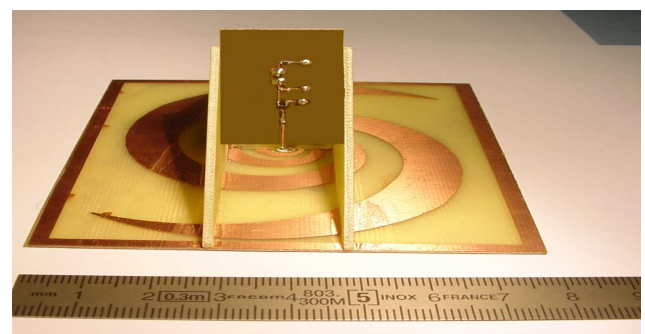

Fig.15 Rectenna prototype

The Dc power harvested with broadband system is very low. The level of RF energy and the mismatching of the antenna to rectifier are the causes of this low level DC power. To increase the DC power harvested and the efficiency of conversion RF/DC we can use the special RF source to feed the devices (WPT). The use of antenna arrays can increase the RF power and the DC power but for attended the significant DC level the size of the array become very large.

\section{Narrow band system:}

As seen in the section I, the maximum of the power density in the urban environment has been measured in the $1.8 \mathrm{GHz}-$ $1.9 \mathrm{GHz}$ band. This power density is $-14.5 \mathrm{dBm} / \mathrm{m}^{2}$ and is constant over time. It is approximately equal to half of the total power density (Fig. 16)

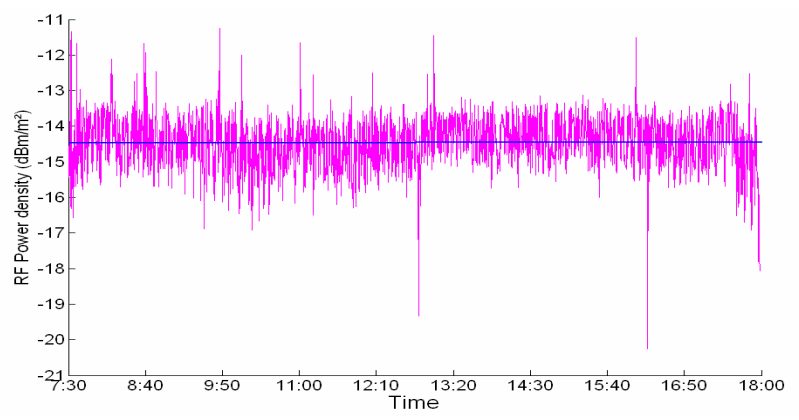

Fig.16: Total measured RF power density as time $(1.8 \mathrm{GHz}-$ $1.9 \mathrm{GHz})$

In this section we present only the results of simulations. For the narrowband the matching circuit is essay to achieve. It is presented in Fig.17. The return loss of the rectifier is presented in Fig. 18.

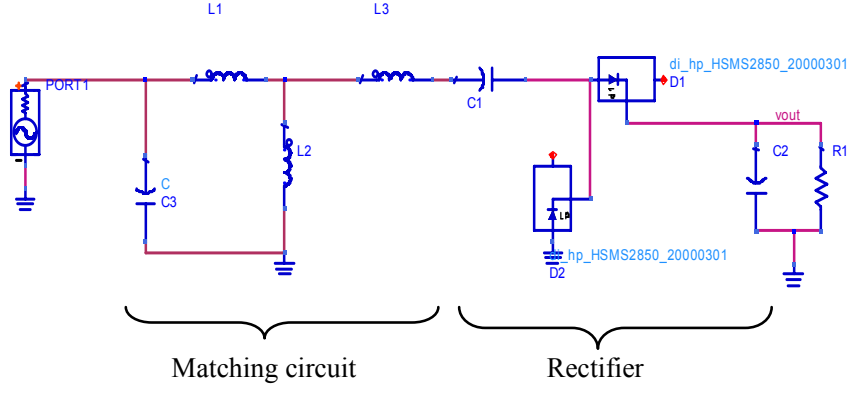

Fig.17: Schema of the rectifier with matching circuit

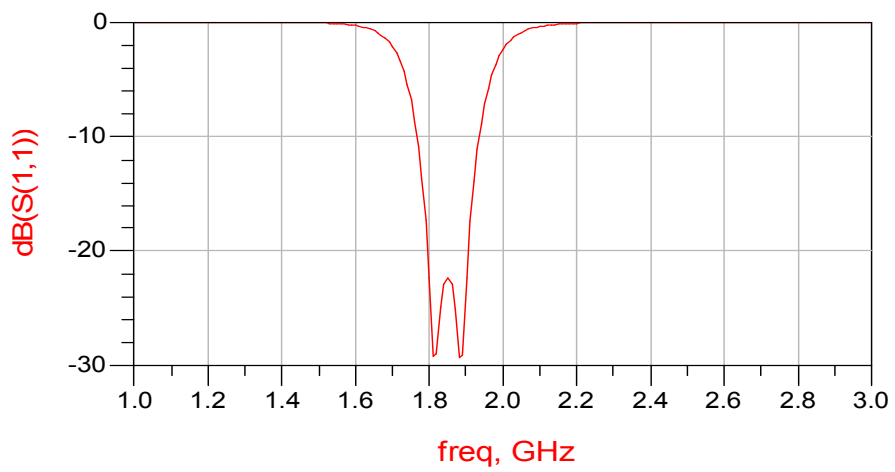

Fig.18. Rectifier return loss 
The estimate DC power scavenged with the narrow band system is presented in Fig.19. The input RF power recovered with antenna is estimated around $-42 \mathrm{dBm}$ $(63 \mathrm{nW})$. The efficiency of rectifier is estimated in order of $0.6 \%$ (Fig.20). The DC power can be attended the $400 \mathrm{pW}$.

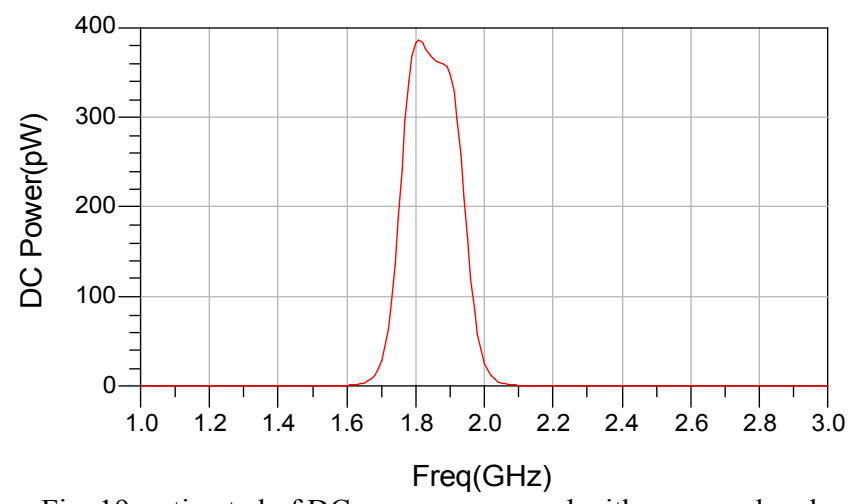

Fig. 19: estimated of DC power scavenged with a narrowband system

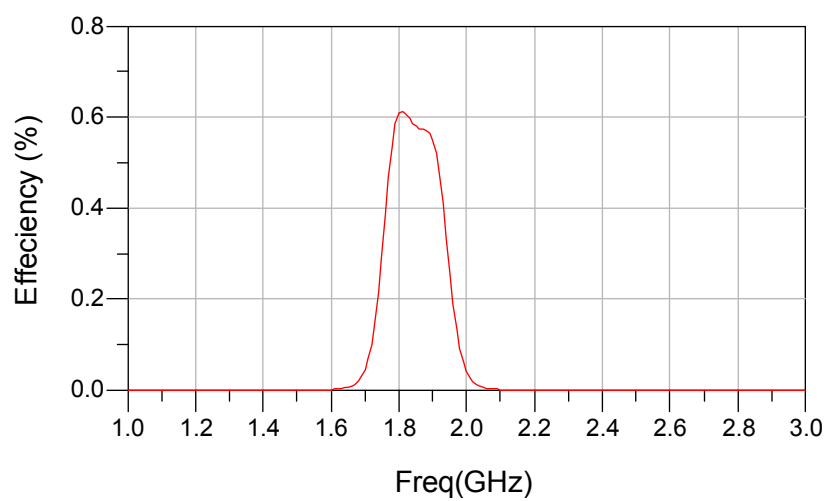

Fig. 20: Estimated efficiency as function frequencies

The use of the matching circuit has increased the DC power. But the efficiency of conversion RF/DC is very low $(0.6 \%)$. This is due to characteristics of the diodes at low power and the voltage junction $\left(\mathrm{V}_{\mathrm{j}}=0.35 \mathrm{~V}\right.$ for HSMS2850). To increase the DC power harvested and the efficiency of conversion RF/DC we should be use the diodes more sensitive than HSMS2850 with a junction voltage near $0 \mathrm{~V}$ and use an antenna arrays to increase the input RF power.

\section{Conclusion:}

In this paper we have presented a study of feasibility to harvesting the ambient RF energy. The measurement of the RF power density available in urban environment shows the RF power is very low and is distributed in a large wide band frequency. To scavenge a maximum of DC power we have presented a wideband system when able to deliver a DC power around $12.5 \mathrm{pW}$. A narrowband system is also presented. The first study for this system show the attended DC power can be about a 400pW.
For the two systems the scavenged DC power is very low to ensure autonomous operation of devices. But this energy harvesting can be store in micro-battery or super capacity. To increase the DC power scavenged we can increase the $\mathrm{RF}$ input power by using for example an antenna arrays.

\section{Reference:}

[1] S. P. Beeby, M. J. Tudor and N. M. White "Energy harvesting vibration sources for microsystems applications" MEASUREMENT SCIENCE AND TECHNOLOGY, Vol. 17, 2006 R175-R195

[2] Brown, W. Mims, J. Heenan, N. "An experimental microwave-powered helicopter" Raytheon Company, Burlington, MA, USA; 1965 IEEE International Record, vol. 13, part 5, pp. 225-235.

[3] R. M. Dickinson, "Evaluation of a microwave high-power reception-conversion array for wireless power transmission," Jet Propulsion Laboratory, California Institute of Technology, Pasadena, CA, Tech. Memo 33-741, Sept. 1975.

[4] Hitoshi Hayami, Masao Nakamura, and Kanji Yoshioka "The Life Cycle CO2 Emission Performance of the DOE/NASA Solar Power Satellite System: A Comparison of Alternative Power Generation Systems in Japan" IEEE TRANSACTIONS ON SYSTEMS, MAN, AND CYBERNETICS PART C: APPLICATIONS AND REVIEWS, VOL. 35, NO. 3, AUGUST 2005

[5] East, T.W.R.; "Self-steering. self-focussing phased array for SHARP" Antennas and Propagation Society International Symposium, 1991. AP-S. Digest 24-28 June 1991 Page(s):1732 1735 vol.3

[6]Catherine DEHOLLAIN "adaptation d'impédance à large bande » presses polytechniques et universitaires Romandes, Lausanne 1996.

[7] Ansoft-HFSS High Frequency Structure Simulator 